International Journal of Soft Computing 7 (4): 199-209, 2012

ISSN: 1816-9503

(C) Medwell Journals, 2012

\title{
Color-Texture Segmentation Using ERKFCM-EMD
}

\author{
${ }^{1}$ C. Mythilii and ${ }^{2}$ V. Kavitha \\ ${ }^{1}$ Department of Electrical and Engineering, ${ }^{2}$ Department of Computer Science and Engineering, \\ University College of Engineering, Konam, Nagercoli, Tamilnadu, India
}

\begin{abstract}
A new segmentation approach is proposed in this study which combines color-texture features to get accurate segmentation. The color image is transformed from RGB color space to Lab color space. The statistical color features are extracted from Lab color space. The fuzzy texture unit is determined by the extraction of local texture information from each pixel. The combined feature extraction of color and texture are implemented using Effective Robust Kernelized Fuzzy C-Means clustering strategy (ERKFCM). Finally, refinement processes are used to eliminate the misclassified pixels produced by clustering. It is based upon Earth Mover Distance (EMD). The objective of the study is to measure and analyze Mean Square Error (MSE), Peak Signal to Noise Ratio (PSNR) and Structural SIMilarity (SSIM) by means of color and texture based segmentation and by means of Human Labeled (Ground Truth) Segmentation. Proposed (ERKFCM-EMD) segmentation algorithm is compared quantitatively and qualitatively with K-Means and Fuzzy C-Means Clustering. The input images are obtained from Berkeley databases which are in RGB Color Model. It is concluded that the ERKFCM-EMD Method has outperformed quantitatively and qualitatively results when compared to the existing methods in segmentation.
\end{abstract}

Key words: Color features, texture unit, ERKFCM-EMD, mean square error, peak signal to noise ratio, structural similarity

\section{INTRODUCTION}

Image segmentation plays a vital role in computer vision and image processing applications. In computer vision, segmentation is applied to the process of partitioning a digital image into multiple segments (sets of pixels, also known as super pixels). Image segmentation is typically used to locate objects and boundaries (lines, curves, etc.) in images. More precisely, image segmentation is the process of assigning a label to every pixel in an image so that pixels with the same label share certain visual characteristics. The result of image segmentation is a set of segments that collectively cover the entire image or a set of contours extracted from the image. Each of the pixels in a region is similar with respect to some characteristic or computed property such as color, intensity or texture. Due to the importance of image segmentation a number of algorithms have been proposed by researchers. Based on the input image, segmentation algorithm can be chosen to get the better results (Hassan et al., 2010).

From the survey it is found that the image information obtained as color feature and texture feature when applied individually is not sufficiently robust to accurately describe the image content. In particular the segmentation of natural images has proved to be a challenging task since, these images exhibit significant homogeneities in color and texture (Kothainachiar and Banu, 2007). The strength of texture and color attributes can considerably vary from image to image. Many researchers have solved these problems partially. The color and texture information is most commonly used for image analysis tasks such as object recognition, scene understanding and image retrieval. Color based segmentation provides sharp edges but often in homogeneous regions. At the same time, texture features give us fuzzy boundaries but more homogenous regions. The key solution to address this problem is by combining the color and texture information with an understanding of image characteristics. By adding these two features, sharp boundaries and homogeneous regions are obtained. This is achieved by sequential implementation methodology and the unsupervised classification (Kato and Pong, 2006).

\section{RELATED WORKS}

Zoltan et al. (2003) proposed unsupervised segmentation of color textured images using a multilayer Markov Random Field (MRF) Image Segmentation Model which aims at combining color and texture features. This

Corresponding Author: C. Mythilii, Department of Electrical and Engineering, University College of Engineering, Konam, Nagercoli, Tamilnadu, India 
algorithm has been tested on a variety of synthetic and real image. The computation time was $3-10 \mathrm{~min}$ in Pentium III 933. The combined layer produces slightly higher misclassification rates than the color layer. Zoltan and Ting-Chuen (2006) proposed a Markov Random Field Image Segmentation Model which aims at combining color and texture features (Comaniciu and Meer, 2002). In this study, the perceptually uniform $\mathrm{CIE}-\mathrm{L} \times \mathrm{u} \times \mathrm{v} \times$ color values are considered as color features and a set of Gabor filters as texture features are used. In this study, the implementation is a parallel process. A sequential implementation methodology has been adopted in this approach that has improved the computation speed. Maheswari et al. (2011) has proposed a new approach for color texture segmentation using Haralick features extracted from color co-occurrence matrices, calculated in HIS color space. This study has mainly focused on the unsupervised segmentation of color texture images as a prototypical application in computer vision. Fuzzified distance metric is used to achieve texture segmentation Xiang-Yang et al. (2011) has proposed a new approach for color image segmentation based on Support Vector Machine (SVM) and Fuzzy CMeans (FCM). Here the pixel-level color features and texture features extracted via the Local Homogeneity Model and Gabor filter of the image are the inputs to the SVM Model (classifier). Then, the SVM Model is trained by using FCM with the extracted pixel-level features. Finally the color image is segmented with the trained SVM Model (classifier).

\section{ERKFCM-EMD BASED SEGMENTATION}

The proposed method segments the input image based on color-texture feature extraction. Color is one common feature used in image segmentation. It is often used in conjunction with texture information to achieve better segmentation results. The input image is transformed from RGB to Lab color space. Lab color space is much more intuitive than RGB. Lab Color System approximates human vision and its Luminance component $\mathrm{L}$ and the chrominance component $\mathrm{a}, \mathrm{b}$ matches closely with human perception of light. It has perceptual color fidelity. It has unique color differences sensitivity to a good approximation and is very convenient to measure small color difference while the RGB color space does not (Maheswari et al., 2011). It can be used to make accurate color balance corrections. The color features were extracted from $\mathrm{L}$ and a component based on mean and mode. The $\mathrm{b}$ component doesn't produce significant color composition when compared to that of a. The combined color and texture features are used in Fuzzy

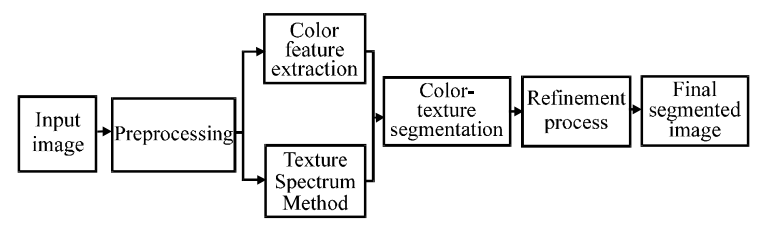

Fig. 1: Block diagram of ERKFCM-EMD based color image segmentation

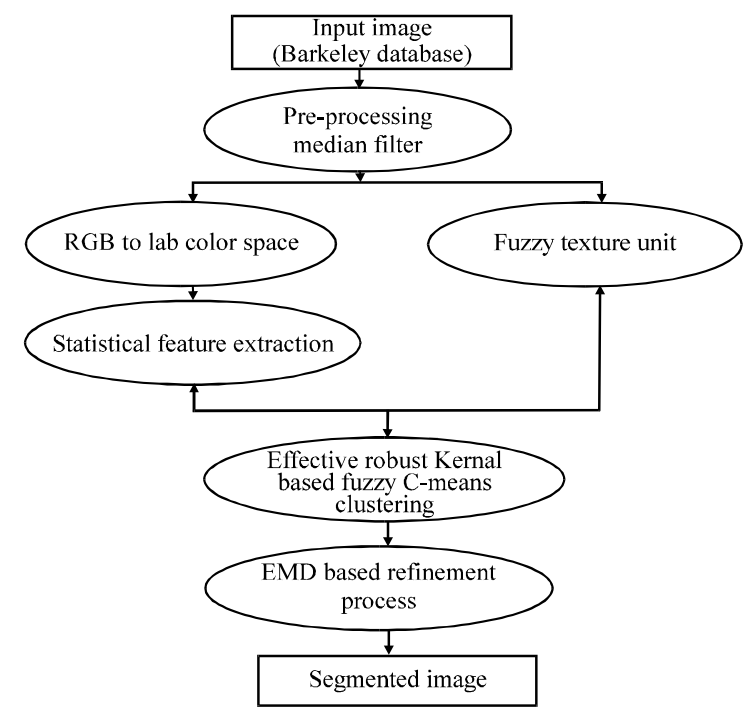

Fig. 2: Work flow of the ERKFCM-EMD segmentation process

C-means and earth mover distance. The block diagram of ERKFCM-EMD color image segmentation and work flow of the proposed method is shown in Fig. 1 and 2.

The input image is pre processed using the median filter to enhance the input image. The image is converted from RGB to Lab color space in order to extract the color statistical features by calculating the mean and mode of $\mathrm{L}$ and a component. The texture features is extracted using fuzzy texture spectrum. The extracted features are segmented with Effective Robust Kernelized Fuzzy C-Means and followed by Earth Mover Distance refinement process to keep an efficient segmentation.

\section{PREPROCESSING}

The image is read and converted into $2 \mathrm{D}$ matrix form. This matrix is the input to the preprocessing stage. The processing involves filtering, normalization, segmentation and object identification. Filtering is one of the most important and usual parts of preprocessing. Three main types of noise exist: impulse noise, additive noise and multiplicative noise. Impulse noise is usually characterized 
by some portion of image pixels that are corrupted, leaving the remaining pixels unchanged. Additive noise occurs when a value from a certain distribution is added to each image pixel, e.g., Gaussian distribution. Multiplicative noise is generally more difficult to remove from images because the intensity of the noise varies with the signal intensity (Schulte et al., 2007). Median filters are good at removing salt and pepper noise from an image (Emre et al., 2007) and also causes relatively little blurring of edges. The median filter is used to remove noise in the pre-processing stage. However, with median filtering, the value of an output pixel is determined by the median of the neighborhood pixels $(3 \times 3)$ rather than the mean. Median filtering is therefore better, to remove these outliers without reducing the sharpness of the image.

\section{FEATURE EXTRACTION}

The feature is defined as a function of one or more measurements, each of which specifies some quantifiable property of an object and is computed such that it quantifies some significant characteristics of the object. The collection of the features of the contents is known as a feature vector. Feature vectors describe particular characteristics of an image based on the nature of the extraction method. The dimension of the vector is determined by the number of features extracted (Choras, 2007). Based upon the literature survey, the features can be classified as follows general and domain features. General features: application independent features such as color, texture and shape. According to the abstraction level, they can be further classified into three categories: pixel-level features are calculated at each pixel, e.g., color, location); local features are calculated over the results of subdivision of the image band on image segmentation or edge detection and global features are calculated over the entire image or just regular sub-area of an image. Domainspecific features: application dependent features such as human faces, fingerprints and conceptual features. These features are often a synthesis of low-level features for a specific domain.

Color feature usually preserves boundaries but it is more sensitive to local color variations. Hence, color based segmentation provides sharp edges but often in homogeneous regions. On the other hand, texture features give us fuzzy boundaries but more in homogeneous regions. The feature based color texture segmentation algorithms were developed by building on the assumption that the separately extracted color and texture features are locally homogeneous and the segmentation task can be viewed as a statistical or probabilistic image partitioning process. The statistical color texture integration schemes are based on K-means, Fuzzy clustering, Neural Networks and Effective Robust Kernel based Fuzzy C-Means clustering. The probabilistic color texture integration includes Bayesian classification, ExpectationMaximization and Markova random fields. The best segmentation results are obtained when the input images are converted to the $\mathrm{CIE}$ lab color representation compared to the other color spaces like, RGB, XYZ, YIQ, YCbCr, I1I2I3, HSV and HIS. The fusion of color (L and a components) and texture features are implemented using an Effective Robust Kernel based Fuzzy C-Means clustering.

Color feature extraction: Color is a widely used important feature for image representation. This is very important as it is invariant with respect to scaling, translation and rotation of an image. Color features are easy to obtain, often directly from the pixels intensities like color histogram over the whole image, over a fixed sub image or over a segmented region. It is one of the most used features in image retrieval. The colors are described by their color space: RGB, LAB, LUV and HSV. RGB is the best known color space which is commonly used for visualization. Color space, color quantification and similarity measurement are the key components of color feature extraction (Ilea and Whelan, 2011). Here CIE LAB color space is selected to extract color features. First, the input image is converted to the CIE Lab color space, prior to the calculation of the statistical color features. The statistical color features are mean and mode (Cao et al., 2010).

Feature extraction can be used to differentiate images based on their features of color. The mean of $3 \times 3$ neighborhood pixels is the average arithmetic intensity value, computed by adding them and dividing the result by their total number. In this study the statistical mean value of an image is calculated based on overlapped $3 \times 3$ window size. It is defined as:

$$
\begin{gathered}
\text { Mean }\left(\mathrm{L}_{\mathrm{Mean} M, \mathrm{~N}}\right)=\frac{\sum_{\mathrm{i}=-1}^{+1} \sum_{\mathrm{j}=-1}^{+1} \mathrm{~L}(\mathrm{M}+\mathrm{i}, \mathrm{N}+\mathrm{j})}{9} \\
0<\mathrm{M}<\text { (Image height }-1) \\
0<\mathrm{N}<(\text { Image width }-1)
\end{gathered}
$$

where, $\mathrm{M}$ and $\mathrm{N}$ are the number of rows and columns in the input image, respectively. In statistics, the mode has the characteristic that occurs most frequently in a data set or a probability distribution:

$$
\begin{aligned}
\text { Mode }\left(\mathrm{L}_{\text {meanM, N }}\right)= & \text { mode }(3 \times 3 \text { neighbourhood } \\
& \text { element of } M, N)
\end{aligned}
$$


$0<\mathrm{M}<$ (Image height - 1)

$0<\mathrm{N}<$ (Image width - 1$)$

where, $\mathrm{M}$ and $\mathrm{N}$ are the number of rows and columns in the input image, respectively. The results for extracting the color feature are shown in Fig. 3. Figure 3a shows the input image. Figure $3 \mathrm{~b}$ shows the lab color image. Figure $3 \mathrm{c}$ and $\mathrm{d}$ show the statistical mean of $\mathrm{L}$ and a components represented in the form of image. Figure $3 \mathrm{e}$ and $\mathrm{f}$ represent the statistical mode of $\mathrm{L}$ and a components represented in the form of image.

Texture Spectrum Method: Texture is another important property of images and they are represented by texels (Victor et al., 2010). The texture spectrum is introduced and described in detail by $\mathrm{He}$ and $\mathrm{Li}$ Wang is the father of

(a) Original image

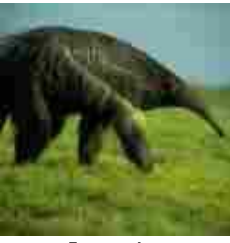

Image 1 (b) Lab color image

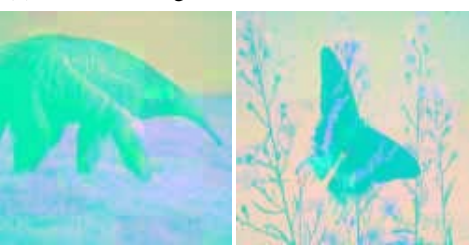

(c) L mean image
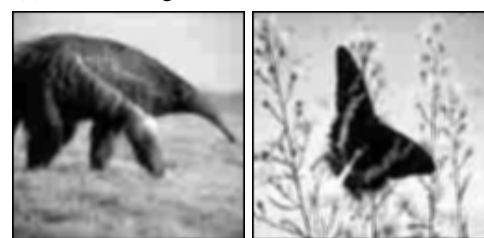

(d) a mean image
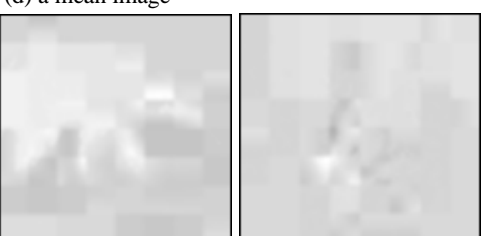

(e) L mode image
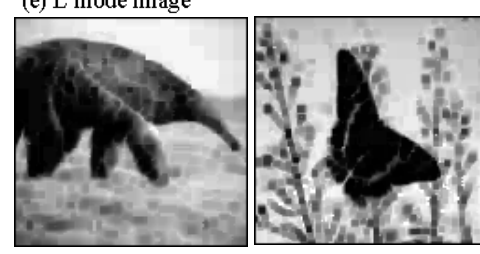

(f) a mode image
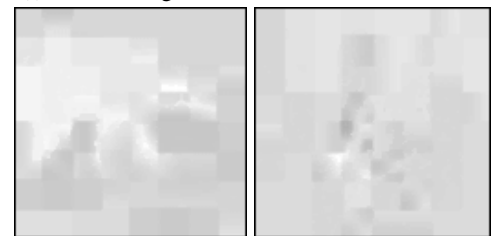
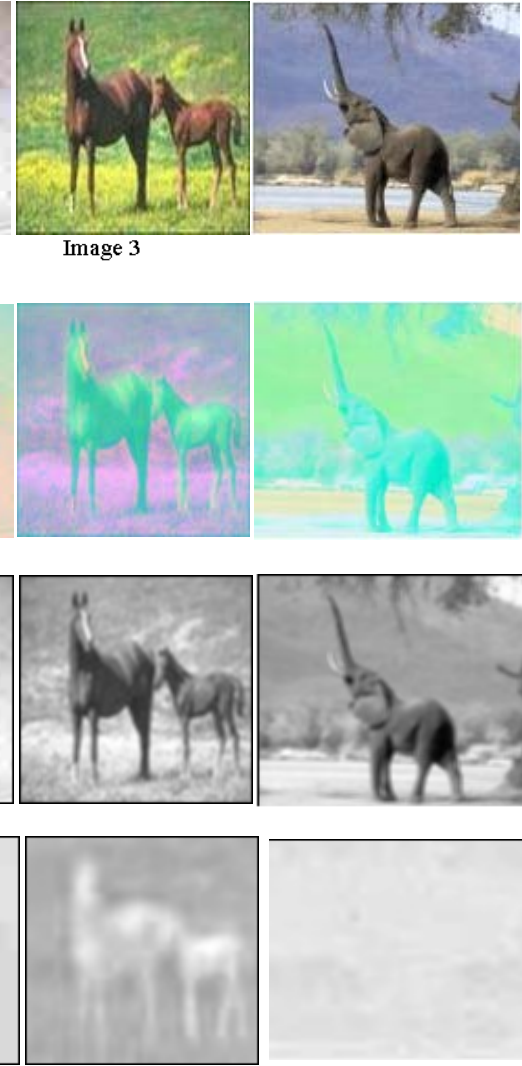

Image 3
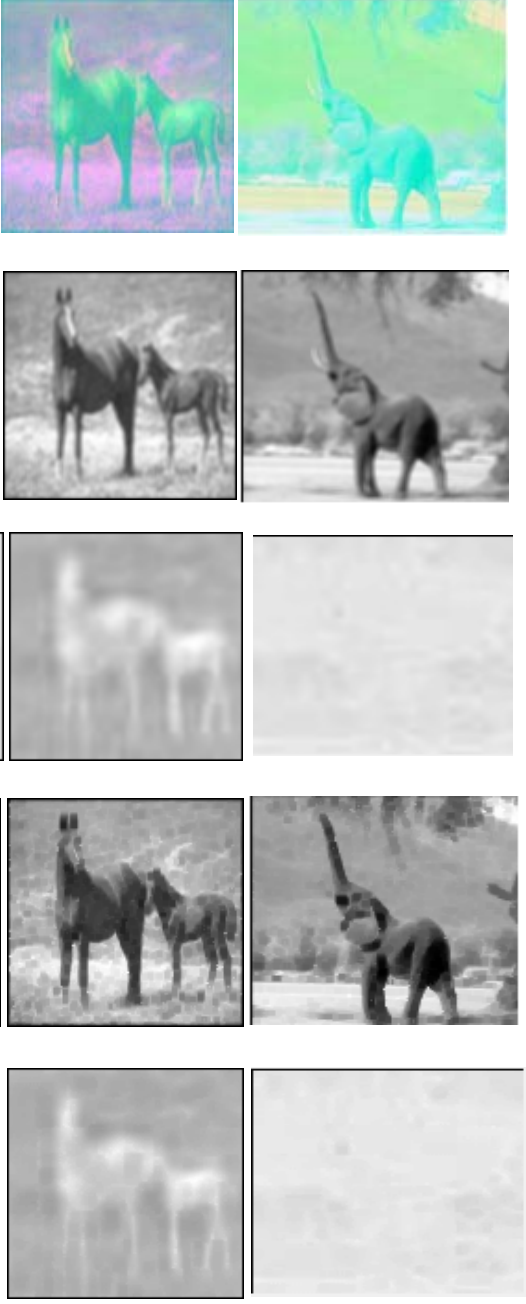

Fig. 3: Color features extraction; a) original image, b) Lab color image, c) L mean image, d) a mean image, e) L mode image and f) a mode image 
Int. J. Soft Comput., 7 (4): 199-209, 2012

\begin{tabular}{|l|l|l|}
\hline $\mathrm{E}_{1}$ & $\mathrm{E}_{2}$ & $\mathrm{E}_{3}$ \\
\hline $\mathrm{E}_{8}$ & & $\mathrm{E}_{4}$ \\
\hline $\mathrm{E}_{7}$ & $\mathrm{E}_{6}$ & $\mathrm{E}_{5}$ \\
\hline
\end{tabular}

Fig. 4: Fuzzy texture unit

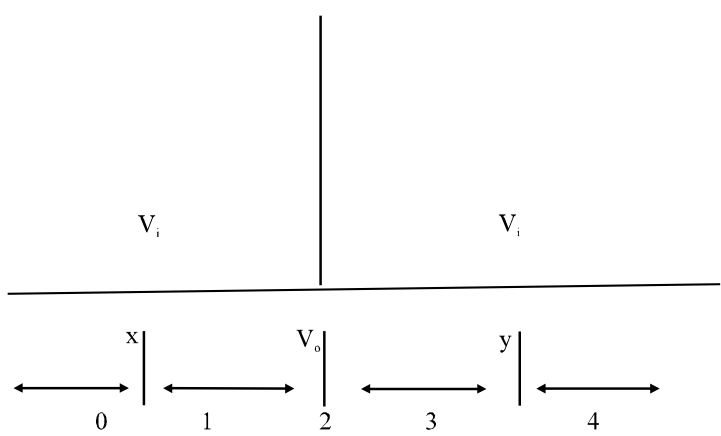

Fig. 5: Texture unit representation

the identification of texture spectrum in an image is achieved by the extraction of local texture information for each pixel. Texture spectrum has been introduced in the past few years (Barcelo et al., 2005; He and Wang, 1990, 1991). The texture image can be decomposed into a set of essential small units, called Texture Units (TU). The occurrence distribution of Texture Units is called as Texture Spectrum (TS) the basic concept is that a texture image can be considered as a set of essential small units termed as texture units which characterize the local texture information for a given pixel and its neighborhood. In a square-raster digital image, each pixel is surrounded by eight neighboring pixels. The local texture information for a pixel can be extracted from a neighborhood of $3 \times 3$ pixels which represents the smallest complete unit (in the sense of having eight directions surrounding the pixel). The ordering of elements and representation of texture unit are shown in Fig. 4 and 5 .

A neighborhood of $3 \times 3$ pixels is denoted by a set containing nine elements: $\mathrm{V}=\left\{\mathrm{V}_{0}, \mathrm{~V}_{1}, \mathrm{~V}_{2}, \ldots, \mathrm{V}_{8}\right\}$ where $V_{0}$ represents the intensity value of the central pixel and $V_{i}(1 \leq i \leq 8)$ the intensity value of each neighboring pixel (Jiji and Ganesan, 2010). Then, the corresponding texture unit can be represented by $\mathrm{TU}=\left\{\mathrm{E}_{1}, \mathrm{E}_{2}, \ldots \mathrm{E}_{8}\right\}:$ (a)

\begin{tabular}{|r|r|r|}
\hline 80 & 110 & 130 \\
\hline 150 & 120 & 180 \\
\hline 90 & 120 & 200 \\
\hline
\end{tabular}

(b)

\begin{tabular}{|l|l|l|}
\hline 0 & 1 & 3 \\
\hline 4 & & 4 \\
\hline 0 & 2 & 4 \\
\hline
\end{tabular}

Fig. 6: a) $3 \times 3$ neighborhood and b) Texture Unit TU number

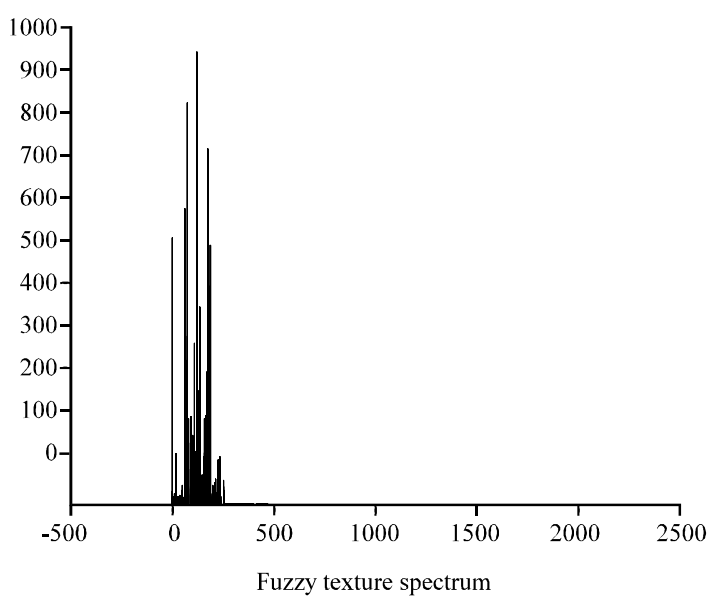

Fig. 7: Color fuzzy texture spectrum (Image 1)

Where:

$$
\mathrm{E}_{\mathrm{i}}=\left\{\begin{array}{l}
0 \text { if } \mathrm{V}_{\mathrm{i}}<\mathrm{V}_{0} \text { and } \mathrm{V}_{\mathrm{i}} \leq \mathrm{X} \\
1 \text { if } \mathrm{V}_{\mathrm{i}}<\mathrm{V}_{0} \text { and } \mathrm{V}_{\mathrm{i}}>\mathrm{X} \\
2 \mathrm{~V}_{\mathrm{i}}=\mathrm{V}_{0} \text { for } \mathrm{i}=1,2, \ldots 8 \\
3 \text { if } \mathrm{V}_{\mathrm{i}}>\mathrm{V}_{0} \text { and } \mathrm{V}_{\mathrm{i}} \leq \mathrm{Y} \\
4 \text { if } \mathrm{V}_{\mathrm{i}}>\mathrm{V}_{0} \text { and } \mathrm{V}_{\mathrm{i}}>\mathrm{Y}
\end{array}\right\}
$$

where $\mathrm{x}, \mathrm{y}$ are user-specified values. As each element of TU has one of the three possible values, the combination of all the eight elements results in $3^{8}=6561$ possible texture units in total. The 6561 texture units are labeled by using the following Eq. 3 and 4:

$$
\begin{aligned}
& \mathrm{N}_{\mathrm{TU}}=\sum_{\mathrm{i}=1}^{8} \mathrm{Ei} \times 5^{\frac{(\mathrm{i}-1)}{2}} \\
& \mathrm{~N}_{\mathrm{TU}} \varepsilon\left\{0,1 \ldots\left(3^{8}-1\right)\right\}
\end{aligned}
$$

NTU represents the texture unit number and $\mathrm{Ei}$ is the ith element of texture unit set, $\mathrm{TU}=\{\mathrm{E} 1, \mathrm{E} 2, \ldots, \mathrm{E} 8\}$. The size of the window depends on the nature of the texture image and the process of formation of a texture unit is shown in Fig. 6a and b. For example: 


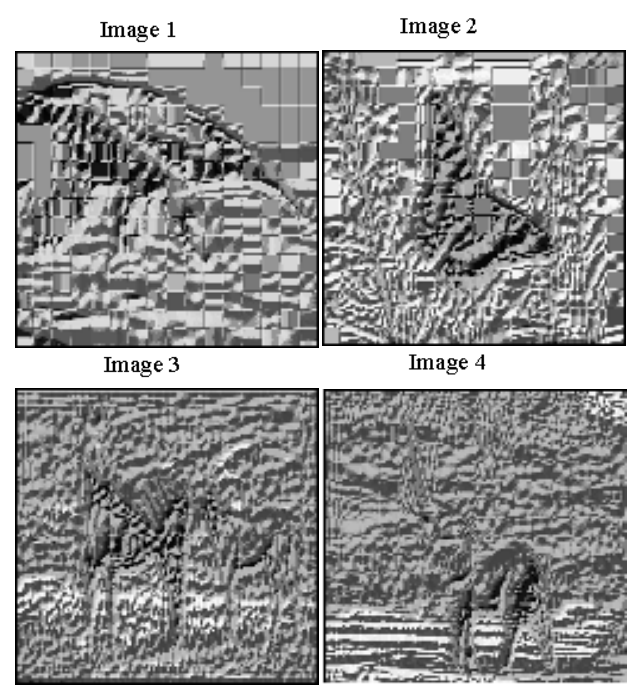

Fig. 8: Color texture unit for samples images

$$
\begin{gathered}
\mathrm{V}=\{80,110,130,180,200,120,90,150\} \\
\mathrm{TU}=\{1,3,4,4,2,0,4\}
\end{gathered}
$$

The given image color fuzzy texture spectrum and the corresponding color texture unit image are shown in Fig. 7 and 8 . This proposed color fuzzy texture unit image is used for color texture segmentation.

\section{EFFECTIVE ROBUST KERNELIZED FUZZY C-MEANS (ERKFCM)}

The combined feature extraction of color and texture are implemented using Effective Robust Kernelized Fuzzy C-means clustering strategy (Kannan et al., 2010). The widely used FCM for image segmentation has some limitations because of its squared-norm distance, measure to measure the similarity between centers and data objects of images which are corrupted due to heavy noise and outliers and also clustering performance are affected by the initial center and clusters. To overcome the above limitations ERKFCM function is used based on kernel function and effective initialization. It includes robust non-Euclidean distance measures using properties of kernel function. The composition steps of algorithm are:

Algorithm 1: ERKFCM based segmentation.

Input: Color image.

Step 1: Extract the color and texture features from the input image.

Step 2: Choose the number of clusters and assign the initial cluster centers using center initialization algorithm.

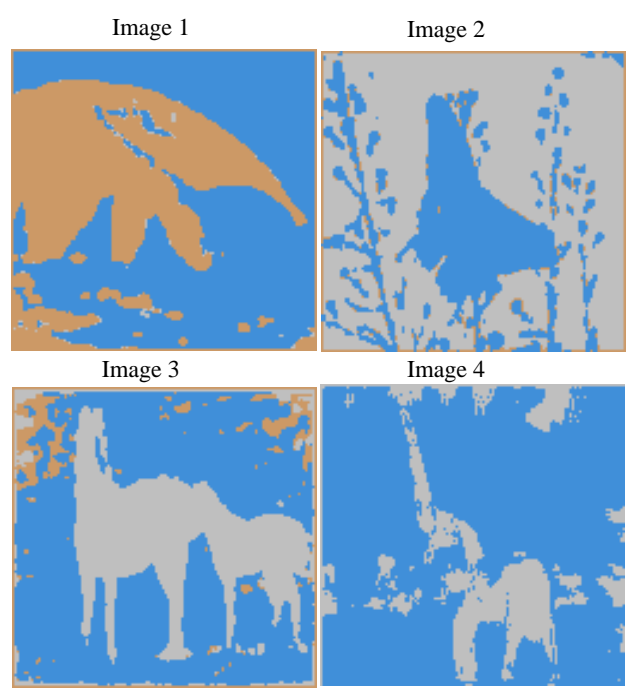

Fig. 9: The results of ERKFCM

Step 3: Compute partition matrix $\mathrm{U}_{\mathrm{ij}}$ using Eq. 5.

Where:

$$
\mathrm{U}_{\mathrm{ij}}=\frac{\left[\frac{1}{\varepsilon_{2}-\mathrm{K}\left(\mathrm{X}_{\mathrm{i}}, \mathrm{V}_{\mathrm{j}}\right)}\right]^{\frac{1}{\mathrm{~m}-1}}}{\sum_{\mathrm{i}=1}^{\mathrm{c}}\left[\frac{1}{\varepsilon_{2}-\mathrm{K}\left(\mathrm{X}_{\mathrm{i}}, \mathrm{V}_{\mathrm{j}}\right)}\right]^{\frac{1}{\mathrm{~m}-1}}}
$$

$\mathrm{X}_{\mathrm{i}} \quad=$ Object

$\mathrm{V}_{\mathrm{j}} \quad=$ Center

$\mathrm{K}\left(\mathrm{X}_{\mathrm{i}}, \mathrm{V}_{\mathrm{j}}\right)=$ Kernel function-exponent value

$\mathrm{c} \quad=$ No. of clusters

$\varepsilon_{2} \quad=$ The parameter which can be adjusted by user

Step 4: Update the cluster center using Eq. 6:

$$
V_{j}=\frac{\sum_{i=1}^{N} U_{i j}^{m} X_{i}}{\sum_{i=1}^{N} U_{i j}^{m}}
$$

Step 5: Repeat steps 3-4 until the following termination criterion is satisfied. Where $\left\|V^{\text {present }}-V^{\text {prenious }}\right\|<\varepsilon$. Where $\mathrm{V}^{\text {present }}$ and $\mathrm{V}^{\text {previous }}$ are the vector of cluster prototypes at the present and previous iteration. The output of ERKFCM shown in Fig. 9.

\section{REFINEMENT RPOCESS}

In this refinement process, cross-bin metric such as Earth Mover's Distance (EMD) is used to enhance the segmentation process. The main aim of this research is to segment the given dynamic scene into foreground and background regions. Segmentation based on gray level histogram thresholding is a method to divide an 


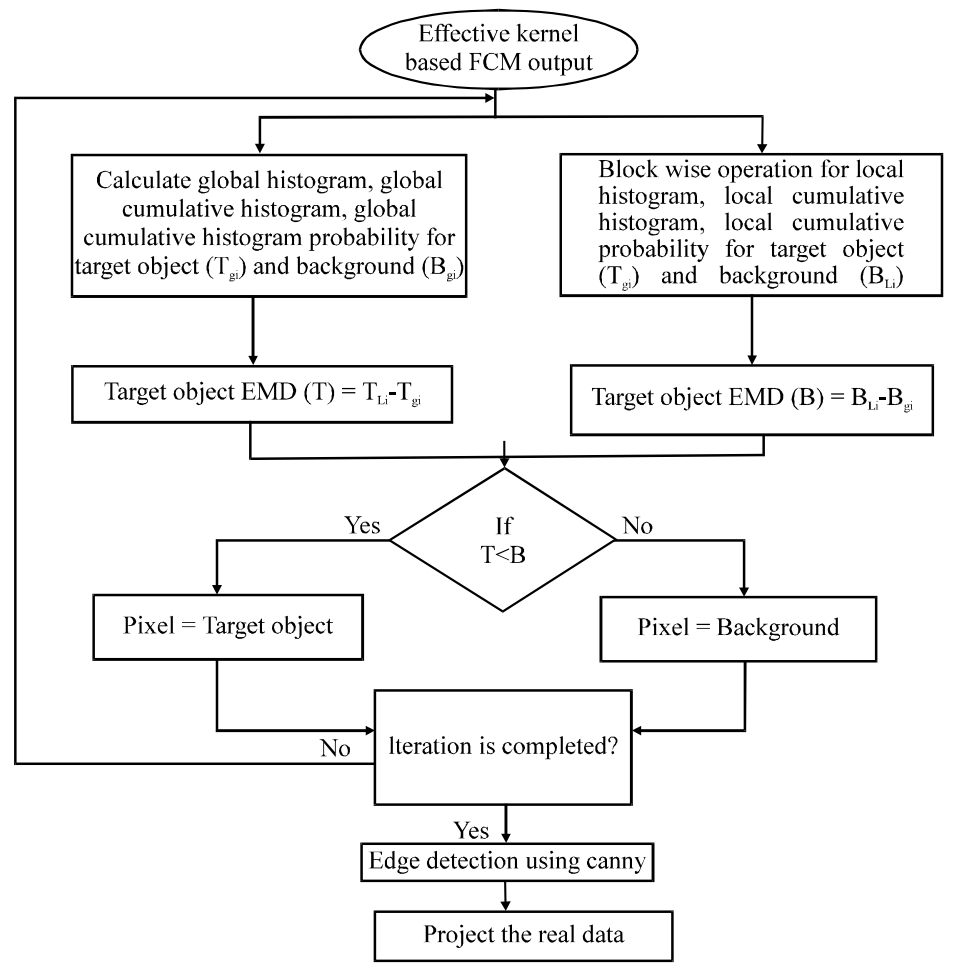

Fig. 10: Earth Mover Distance (EMD) refinement process

image containing two regions of interest: object and background. In fact applying this threshold to the whole image pixels whose gray level is under the threshold valve are assigned to a region and the remainder to the curve (Adam and Kimmel, 2009). There are two level of implementation in this study. In the first level ERKFCM based image segmentation is performed and in the second level the EMD based refinement is done. In the second stage it uses curve evolution variation framework for segmentation. The flow fields driving the curves are based on the distribution of features in the inner and outer regions bounded by curves. Here curve evolution is based on histogram of images. The flow fields are derived to guide the evaluation process based on EMD for measuring the dissimilarity between two histograms (Adam and Kimmel, 2009). It is shown in Fig. 10.

\section{Algorithm 2 (EMD refinement process)}

Input: Segmented output from Effective kernel based Fuzzy C-Means clustering.

Step 1: Calculate the global histogram, global cumulative histogram and probability for Target object $\mathrm{T}_{\mathrm{gi}}$ and background $\mathrm{B}_{\mathrm{g} \text {. }}$.

Step 2: Calculate the local histogram, local cumulative histogram and probability for Target object $\mathrm{T}_{\mathrm{Li}}$ and Background $_{\mathrm{L} \text {. }}$.
Step 3: To find the target object $T=T_{\mathrm{li}} \times T_{\mathrm{gi}}$ and $\mathrm{B}=\mathrm{B}_{\mathrm{li}} \times \mathrm{B}_{\mathrm{g}:}$.

Step 4: If $T$ is less than $B$, target object is obtained.

Step 5: Edge detection is done on the target object using canny operator.

Step 6: Project the enhanced segmented image.

\section{PERFORMANCE MEASURE}

The proposed method has been used to segment an image into distinct color-texture regions on the Berkeley database. The proposed algorithm was applied to 200 images and the output was compared with the human perceptual ground truth. The Mean Square Error (MSE), Peak Signal to Noise Ratio (PSNR) and Structural Similarity (SSIM) are the three metrics used to compare image segmentation quality.

Mean Square Error (MSE): It represents the cumulative squared error. MSE value is the difference between the segmented images and the ground truth image (Xiang-Yang et al., 2011). The lower the value of MSE the error is minimum. The MSE is calculated by Eq. 7 . 


$$
\mathrm{MSE}=\frac{\sum_{\mathrm{m}=1} \sum_{\mathrm{n}=1}\left(\mathrm{I}_{1}(\mathrm{~m}, \mathrm{n})-\mathrm{I}_{2}(\mathrm{~m}, \mathrm{n})\right)^{2}}{\mathrm{M} \times \mathrm{N}}
$$

Where:

$\mathrm{I}_{1} \quad=$ The ground truth image

$\mathrm{I}_{2} \quad=$ The segmented image

$\mathrm{M}$ and $\mathrm{N}=$ The number of rows and columns in the input image, respectively $\left(\mathrm{I}_{1}(\mathrm{~m}, \mathrm{n})-\mathrm{I}_{2}(\mathrm{~m}, \mathrm{n})\right)$

Peak Signal to Noise Ratio (PSNR): It computes the peak signal to noise ratio in decibels between two images. This ratio is often used as a quality measurement between the segmented and ground truth image. The higher the PSNR, the quality of the segmented image is better (Fig. 11). It is calculated by using Eq. 8:

$$
\operatorname{PSNR}=20 \log _{10}\left(\frac{2^{\mathrm{n}}-1}{\sqrt{\mathrm{MSN}}}\right) \mathrm{db}
$$

where, $\mathrm{n}$ is the number of bits used in representing the pixel of the image. For grayscale image, $\mathrm{n}$ is 8 .

Structural SIMilarity (SSIM): It is a method of measuring the similarity between two images. The SSIM

Table 1: Performance evaluation of ERKFCM-EMD segmentation

\begin{tabular}{|c|c|c|c|c|c|c|c|c|c|}
\hline \multirow[b]{2}{*}{$\begin{array}{l}\text { Test } \\
\text { image }\end{array}$} & \multicolumn{3}{|l|}{ PSNR } & \multicolumn{3}{|l|}{ MSE } & \multicolumn{3}{|l|}{ SSIM } \\
\hline & $\begin{array}{l}\text { K-means } \\
\text { clustering }\end{array}$ & $\begin{array}{c}\text { Fuzzy C means } \\
\text { clustering }\end{array}$ & $\begin{array}{l}\text { ERKFCM- } \\
\text { EMD }\end{array}$ & $\begin{array}{l}\text { K-means } \\
\text { clustering }\end{array}$ & $\begin{array}{c}\text { Fuzzy C means } \\
\text { clustering }\end{array}$ & $\begin{array}{l}\text { ERKFCM- } \\
\text { EMD }\end{array}$ & $\begin{array}{l}\text { K-means } \\
\text { clustering }\end{array}$ & $\begin{array}{c}\text { Fuzzy C means } \\
\text { clustering }\end{array}$ & $\begin{array}{l}\text { ERKFCM- } \\
\text { EMD }\end{array}$ \\
\hline$\overline{1}$ & 53.252 & 52.957 & 90.275 & 0.307 & 0.329 & 0.005 & 0.99400 & 0.894 & 0.999 \\
\hline 2 & 56.663 & 49.844 & 71.136 & 0.140 & 0.673 & 0.005 & 0.88400 & 0.988 & 0.999 \\
\hline 3 & 49.341 & 50.544 & 53.857 & 0.756 & 0.573 & 0.267 & 0.96339 & 0.955 & 0.975 \\
\hline 4 & 49.988 & 52.798 & 56.369 & 0.651 & 0.341 & 0.150 & 0.88800 & 0.960 & 0.967 \\
\hline
\end{tabular}

Quantitative evaluation
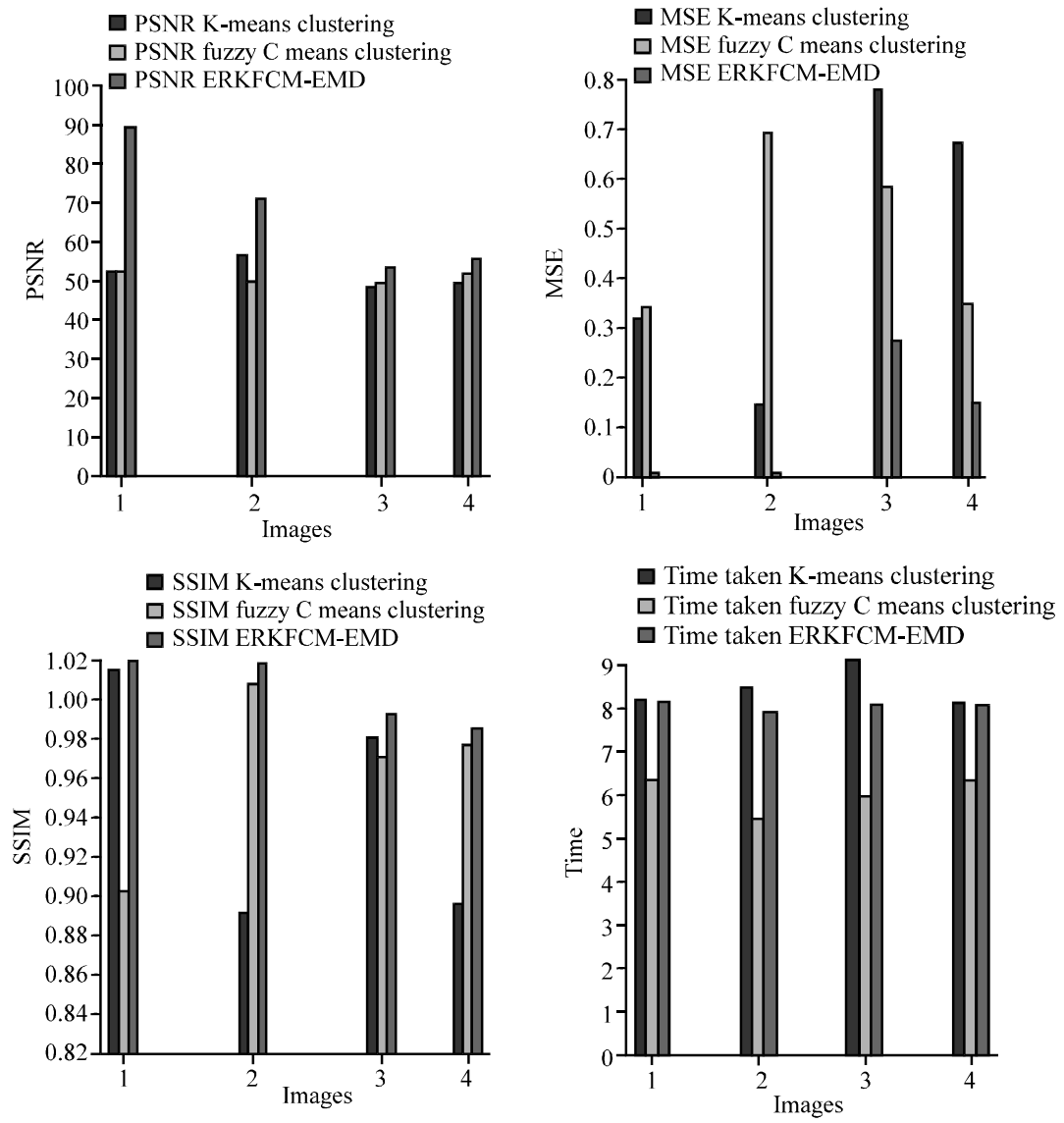

Fig. 11: Graphical representation of MSE, PSNR, SSIM and time for K-means, fuzzy C-means and ERKFCM-EMD 
index is a full reference metric or in other words, the measuring of image quality based on an initial uncompressed or distortion-free image as reference. SSIM is designed to improve on traditional methods like Peak Signal to Noise Ratio (PSNR) and Mean Squared Error (MSE) which has proved to be inconsistent with human eye perception. The measure between two images of common size $\mathrm{N} \times \mathrm{N}$ is:

$$
\operatorname{SSIM}_{\mathrm{x}, \mathrm{y}}=\frac{\left(2 \mu_{\mathrm{x}} \mu_{\mathrm{y}}+\mathrm{C}_{1}\right)\left(2 \sigma_{\mathrm{xy}}+\mathrm{C}_{2}\right)}{\left(\mu_{\mathrm{x}}^{2}+\mu_{\mathrm{y}}^{2}+\mathrm{C}_{1}\right)\left(\sigma_{\mathrm{x}}^{2}+\sigma_{\mathrm{y}}^{2}+\mathrm{C}_{2}\right)}
$$

Where:

$\mu_{\mathrm{x}}=$ The average of $\mathrm{x}$

$\mu_{\mathrm{y}}=$ The average of $\mathrm{y}$

$\sigma_{\mathrm{x}}{ }^{2}=$ The variance of $\mathrm{x}$

$\sigma_{\mathrm{y}}{ }^{2}=$ The variance of $\mathrm{y}$

$\sigma_{\mathrm{xy}}=$ The covariance of $\mathrm{x}$ and $\mathrm{y}$
$\mathrm{C}_{1}=\mathrm{K}_{1} \mathrm{~L}^{2}$,

$\mathrm{C}_{2}=\mathrm{K}_{2} \mathrm{~L}^{2}=$ Two variables to stabilize the division with weak denominator

$\mathrm{L}=$ The dynamic range of the pixel-values $\mathrm{K}_{1}=$ 0.01 and $\mathrm{K}_{2}=0.03$ by default

The difference with respect to other measures mentioned previously such as MSE or PSNR, these measures estimate only the perceived errors, on the other hand SSIM considers image degradation as perceived change in the structural information. Structural information is the perception that the pixels have strong inter-dependencies especially when they are spatially close. These dependencies carry important information about the structure of the objects in the visual scene. The performance evaluations for the three different image segmentation K-means clustering, fuzzy $\mathrm{C}$ means clustering and ERKFCM-EMD are shown in Table 1. The

(a) Original image
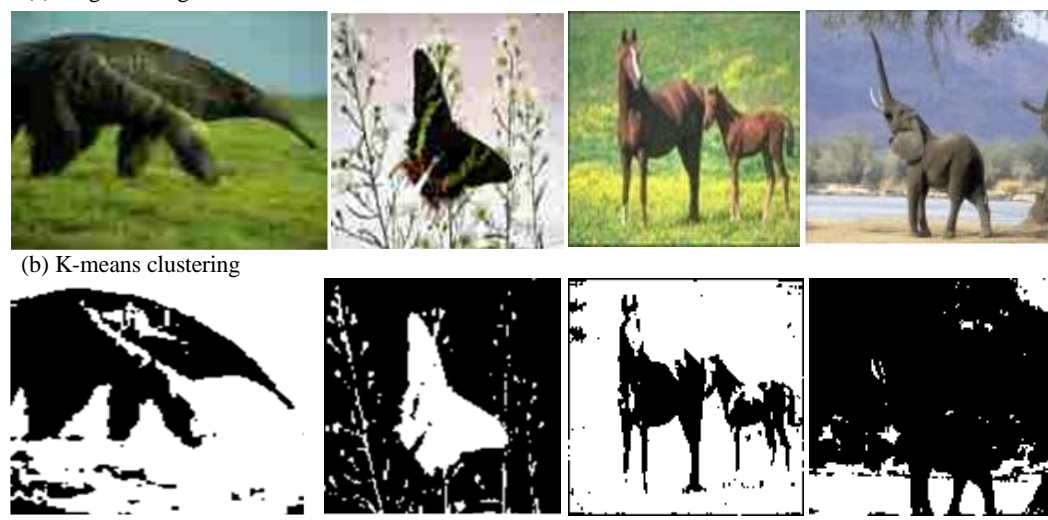

(c) Fuzzy C means clustering
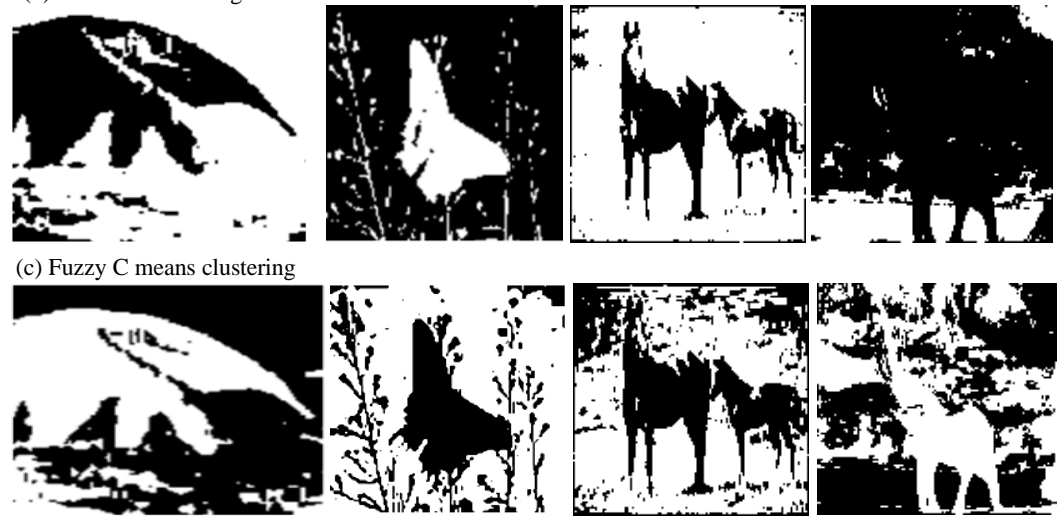

(d) ERKFCM-END Method
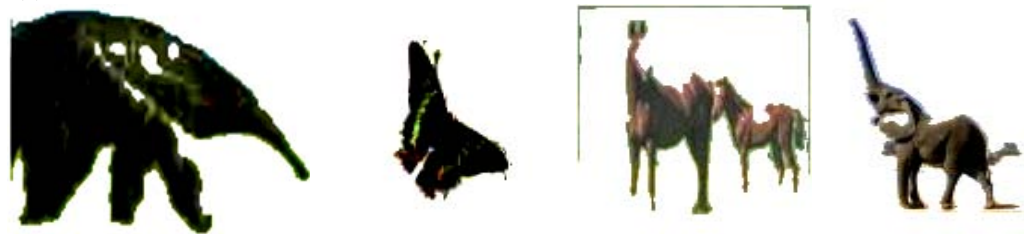

(e) Human labeled segmentation
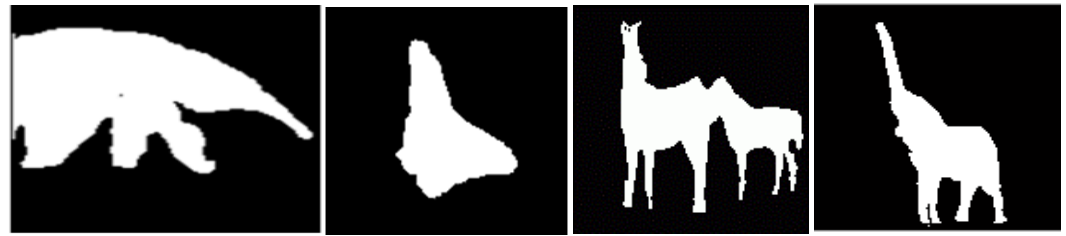

Fig. 12: The comparative results of different segmentation method and human-labeled segmentation 
(a) Original image
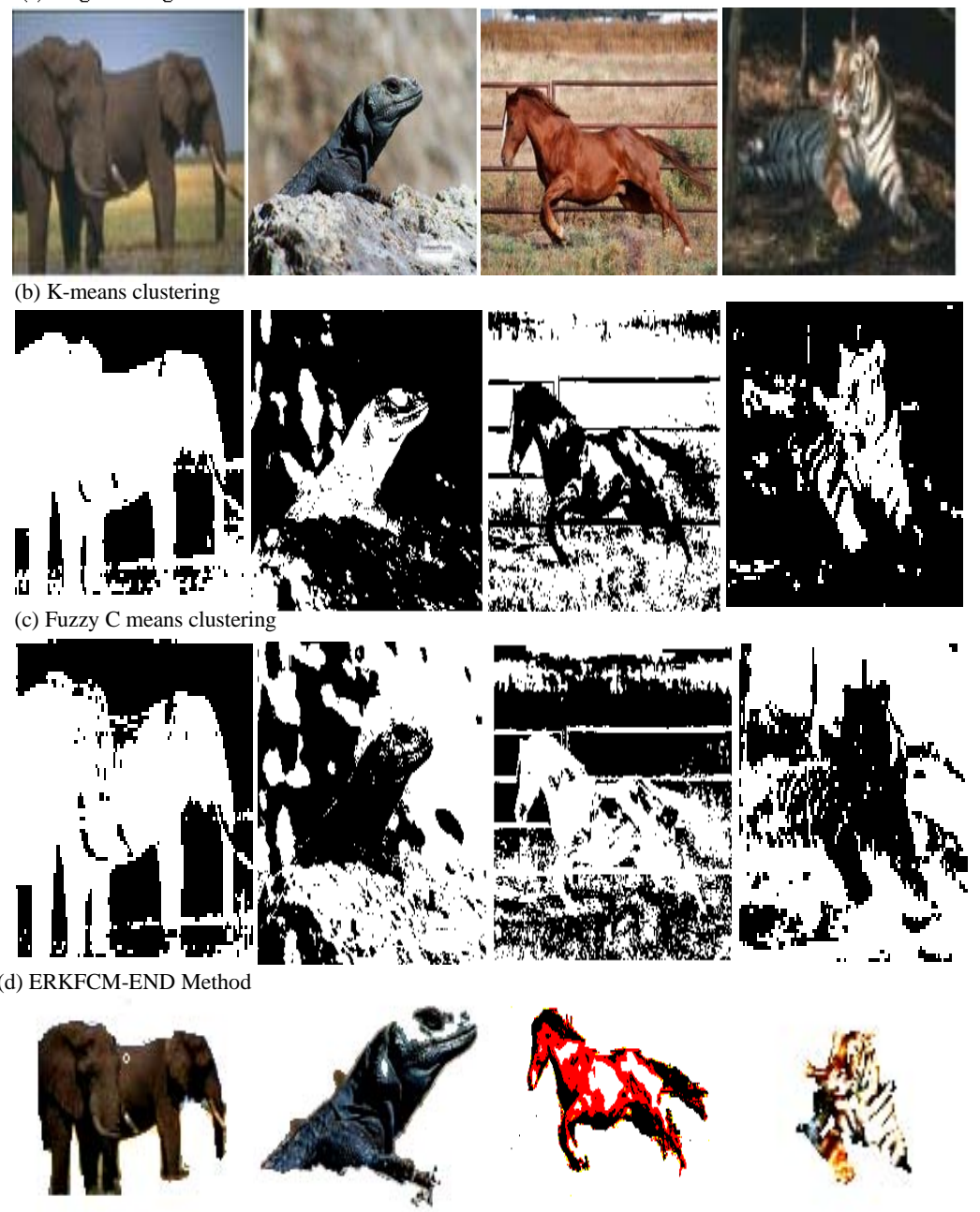

ERKFCM-END Method
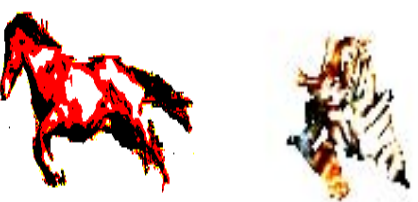

Fig. 13: The comparative results of different segmentation method

Table 2: Time taken of ERKFCM-EMD segmentation

\begin{tabular}{lccc}
\hline & Time taken & & \\
Test & - & & \\
image & K-means clustering & Fuzzy C means clustering & ERKFCM-EMD \\
\hline 1 & 7.3 & 5.7 & 7.3 \\
2 & 7.6 & 4.9 & 7.1 \\
3 & 8.2 & 5.4 & 7.2 \\
4 & 7.3 & 7.2 & 5.7 \\
\hline
\end{tabular}

comparison is performed on the segmentation results of the proposed method on natural images obtained from Berkeley database with K-means clustering and fuzzy $\mathrm{C}$ means clustering. Figure 12 shows the comparative results of different segmentation methods with humanlabeled segmentation (Xiang-Yang et al., 2010). Figure 13 shows segmentation results for few more sample so fimages. The time taken for the above methods is shown in Table 2. It is inferred that the time taken by the proposed method is negligible when compared to that of $\mathrm{K}$-means clustering. The time taken by fuzzy C-means is less but the proposed process involves fuzzy C-means as one of the process in the Effectivem Robust Kernelized Fuzzy C-Means Clustering Method. The time taken by the proposed method is acceptable because refinement is done over segmentation process. The graphical representation of MSE, PSNR. SSIM and Time for Kmeans, Fuzzy C-means and ERKFCM-EMD is shown in Fig. 11

\section{CONCLUSION}

Image segmentation is an important task for computer vision applications. In this study, a new approach has been presented for color image segmentation based on color-texture features. The color features are extracted from statistical characteristics. Then, texture feature is obtained from the fuzzy texture unit. Finally the color image is segmented using effective robust kernelized 
fuzzy $\mathrm{C}$ means clustering and refinement is done based on EMD. Results obtained from the Berkeley database indicate that the proposed technique ERKFCM-EMD has produced better quantitative results than the other state of the art segmentation methods recently proposed in the literature. Drawback of the proposed method is that the time taken for segmentation is not much reduced. The aim of the future research will be towards the focus of optimization in time.

\section{REFERENCES}

Adam, A. and R. Kimmel, 2009. On scene segmentation and histogram based curve evolution. IEEE Trans. Pattern Anal. Mach. Intell., 31: 1708-1714.

Barcelo, A., E. Montseny and P. Sobrevilla, 2005. On fuzzy texture spectrum for natural microtextures characterization. EUSFLAT LFA, pp: 685-690.

Cao, W., Y. Yan and S. Li, 2010. Unsupervised color-texture image segmentation based on a new clustering method. J. Next Gen. Infor. Technol., 1: $96-102$.

Choras, R.S., 2007. Image feature extraction techniques and their applications for CBIR and biometrics systems. Int. J. Biol. Biomed. Eng., 1: 6-16.

Comaniciu, D. and P. Meer, 2002. Mean shift: A robust approach toward feature space analysis. IEEE Trans. Patt. Anal. Machine Intel., 24: 603-619.

Emre, M.C., H.A. Kingravi and Y.A. Aslandogan, 2007. Nonlinear vector filtering for impulsive noise removal from color images. J. Electron. Imaging, Vol: 16, $10.1117 / 1.2772639$.

Hassan, El.M., A. Hammouch and D. Aboutajdine, 2010. A color-texture approach based on mutual information for multispectral image classification. J. Multimedia, 5: 481-487.

He, D.C. and L. Wang, 1990. Texture unit, texture spectrum and texture analysis. IEEE Trans. Geosci. Remote Sens., 28: 509-512.

He, D.C. and L. Wang, 1991. Texture features based on texture spectrum. Pattern Recognition, 24: 391-399.
Ilea, D.E. and P.F. Whelan, 2011. Image segmentation based on the Integration of colour-texture descriptors: A review. Pattern Recognit., 44: 2479-2501.

Jiji, G.W. and L. Ganesan, 2010. A new approach for unsupervised segmentation. Applied Soft Comput., 10: 689-693.

Kannan, S.R., S. Ramathilagam, A. Sathya and R. Pandiyarajan, 2010. Effective fuzzy C-means based kernel function in segmenting medical images. Comput. Biol. Med., 40: 572-579.

Kato, Z. and T.C. Pong, 2006. A Markov random field image segmentation model for color textured images. Image Vision Comp., 24: 1103-1114.

Kothainachiar, S. and R.S.D.W. Banu, 2007. A novel image segmentation based on a combination of color and texture features. GVIP J., 7: 45-51.

Maheswari, G.U., K. Ramar, D. Manimegalai and V. Gomathi, 2011. An adaptive region based color texture segmentation using fuzzified distance metric. Applied Soft Comput., 11: 2916-2924.

Schulte, S., V. de Witte and E.E. Kerre, 2007. A fuzzy noise reduction method for color images. IEEE Trans. Image Process., 16: 1425-1436.

Victor, A., Roselin and D.V. Kavitha, 2010. Preferential image segmentation using $\mathrm{J}$ segmentation based in color, shape and texture. Int. J. Eng. Technol., 2: 131-135.

Xiang-Yang, W., T. Wang and B. Judn, 2011. Color image segmentation using pixel wise support vector machine classification. Pattern Recognit., 44: 777-787.

Zoltan, K. and P. Ting-Chuen, 2006. A Markov random field image segmentation model for color textured images. Image Vision Comput., 24: 1103-1114.

Zoltan, K., P. Ting-Chuen and S.G. Qiang, 2003. Unsupervised segmentation of color textured images using a multi layer MRF model. Proceedings of the Image Processing, ICIP, International Conference on 2003, September 14-17th, 2003, Department of Informatics, University Szeged, Hungary, pp: 961-964. 